years of age, growth slows down, the tongue enlarges and a lumbar kyphosis and prominent forehead develop. Based on the findings in this study, overall intellectual functioning was low but showed no evidence of progressive deterioration except for receptive language skills.

\title{
BRAINSTEM AUDITORY RESPONSE TEST
}

\section{BAER IN HIGH RISK INFANTS}

Brainstem auditory responses (BAER) performed on 667 high risk infants from an infant special care unit were evaluated at the Department of Otolaryngology, University of Texas Medical Branch, Galveston, Texas. Infants who failed the test were classified into two groups; those who failed at $30 \mathrm{~dB}$ hearing level and those who failed at $45 \mathrm{~dB}$ hearing level. At follow-up examination in one, three, or six months, 8 ( $1.2 \%)$ had severe sensorineural hearing impairments (since only $50 \%$ returned for follow-up, 2.4\% was a more accurate incidence). Conductive hearing loss was found in $15.7 \%(17 / 108)$ of those who passed $30 \mathrm{~dB}$ level and in $34.3 \%$ $(12 / 34)$ of those who failed. The use of BAER testing at levels less than $45 \mathrm{~dB}$ permitted detection of middle ear disorders. All of the infants who failed at $45 \mathrm{~dB}$ hearing level and had abnormal results at the $3-4$ month follow-up examination had severe sensorineural or moderate to severe mixed hearing losses. For the group that failed at $30 \mathrm{~dB}$ hearing level and were abnormal at follow-up, $80 \%$ had conductive hearing disorders and $20 \%$ had mild sensorineural hearing impairments. Infants enrolled in a parent-infant program for hearing impairment by 6 months of age were referred from the BAER program. (Kramer S J et al. Auditory brainstem responses and clinical follow-up of high-risk infants. Pediatrics March 1989; $\underline{83}: 385-392)$.

COMMENT. The brainstem auditory evoked response test (BAER) is effective in the early detection of hearing impairments in high risk neonates, and the degree and type of hearing loss may be predicted. However, the children who were referred to the BAER program represented only $31 \%$ of the total number of parent-infant program children with congenital hearing impaiment and only $50 \%$ of the children with multiple handicaps. Some of the hearing impaired children entering the parent-infant program at this center during the period of the study were referred from sources other than the BAER program and were much older when enrolled. An infant hearing assessment program for only high risk infants would fail to identify approximately one-half of hearing impaired children.

TAURINE AND BAER MATURATION

A blinded randamized trial of taurine supplementation of preterm infants was conducted at the Department of Pediatrics, University of Texas Southwestern Medical Center, Dallas, and Ross Laboratories, Columbus, Ohio. Infants who received taurine supplementation had more mature brainstem auditory evoked responses with a reduction in the interval between stimulus and response at two different stimulation 